\title{
Discovery and anti-cancer evaluation of two novel non-ATP-competitive FGFR1 inhibitors in non-small-cell lung cancer
}

Jianzhang $\mathrm{Wu}^{1 \dagger}$, Tao Wei ${ }^{1 \dagger}$, Qinqin Tang ${ }^{1}$, Bixia Weng ${ }^{1}$, Wulan $\mathrm{Li}^{2}$, Xin Jiang ${ }^{1}$, Ting Ding ${ }^{3}$, Xiaokun $\mathrm{Li}^{1}$, Guang Liang ${ }^{1}$, Yuepiao Cai ${ }^{1^{*}}$ and Jiansong $\mathrm{Ji}^{1,4^{*}}$

\begin{abstract}
Background: Fibroblast growth factor receptor 1 (FGFR1) is correlated closely with the occurrence and development of lung cancer. FGFR1 kinase inhibitors have exhibited significant therapeutic effects against non-small-cell lung cancer. Recently, non-ATP competitive FGFR1 inhibitors have attracted extensive attention due to their low side effects.

Methods: Caliper Mobility Shift Assay was used for FGFR1 inhibition test and kinase inhibitory mode study. Hoechst staining and Annexin V/PI staining were used to evaluate the cell apoptosis induction. Western blot were then performed to confirm the intracellular FGFR1 inhibition and apoptotic protein expression. Finally, the anti-tumor effect and mechanism of Af23 and Ad23 was evaluated in vivo.

Results: In this study, we designed, synthesized and discovered two novel non-ATP competitive FGFR1 inhibitors, Af23 and $\mathrm{Ad} 23$, using NDGA as a leading compound. They had $\mathrm{IC}_{50}$ values of $0.6 \mu \mathrm{M}$ and $1.4 \mu \mathrm{M}$ against FGFR1 kinase, respectively. The kinase inhibitory assay carried at different ATP concentrations showed that the FGFR1 inhibition mode of both Ad23 and Af23 was non-ATP-competitive. Further, Af23 and Ad23 significantly suppressed FGFR1 phosphorylation and cell proliferation in non-small-cell lung cancer (NSLCLC) H460 cells and induced cell apoptosis. Af23 and Ad23 also showed significant anti-tumor activity in the H460 xenograft mouse model, accompanied with the inhibition of FGFR1, ERK, and AKT phosphorylation without exhibiting toxicity.
\end{abstract}

Conclusions: These results indicate that Ad23 and Af23 are potential agents for the treatment of non-small-cell lung cancer. This work also provides a structural lead for the design of new non-ATP-competitive FGFR1 inhibitors.

Keywords: Fibroblast growth factor receptor 1, Non-small-cell lung cancer, Non-ATP competitive FGFR1 inhibitors, NDGA, Anti-cancer

\section{Background}

Lung cancer is the most common cause of death from cancer worldwide, and non-small-cell lung cancer (NSCLC) accounts for $85 \%$ of the total incidence [1]. Recently, molecular targeted chemotherapy with RTK inhibitors has been used in clinics for the treatment of NSCLC because of the importance of a series of receptor tyrosine kinases (RTKs) in the development of NSCLC [1,2]. Smallmolecule inhibitors of epidermal growth factor receptor

\footnotetext{
*Correspondence: ypcai@126.com; jjctcty@sina.com

${ }^{\dagger}$ Equal contributors

${ }^{1}$ Chemical Biology Research Center, College of Pharmaceutical Sciences,

Wenzhou Medical University, Wenzhou, Zhejiang 325035, China

Full list of author information is available at the end of the article
}

(EGFR), such as gefitinib and erlotinib, have been approved by the U.S. Food and Drug Administration (FDA) for the treatment of NSCLC [3,4]. Unfortunately, NSCLC treatment remains challenging because of some problems, including adverse effects and drug resistance associated with the ATP-competitive kinase inhibition mode of the majority of RTK inhibitors [5-7]. The ATP-binding pocket is highly conserved among members of the kinase family, and it is difficult to find selective agents $[7,8]$. Moreover, the ATP-competitive inhibitors must compete with high intracellular ATP levels leading to a discrepancy between $\mathrm{IC}_{50} \mathrm{~s}$ measured by biochemical versus cellular assays. The non-ATP-competitive inhibitors, called type II or type III inhibitors, offer the possibility of overcoming 
these problems $[7,9]$. Thus, the development of RTK inhibitors that do not compete with ATP is an urgent need for the treatment of NSCLC.

Fibroblast growth factor receptors (FGFRs) belong to the family of RTK superfamily, and FGFRs, especially FGFR1, was reported to be highly related to the development and progress of NSCLC [1,10-17]. Amplification and activating mutations of FGFR1 have been observed in NSCLC [13,14,17], while silencing the expression of FGFR1 by siRNA or pharmacological inhibition of FGFR1 by small molecules suppresses the development of NSCLC $[11,12,16,17]$. These observations make FGFRs increasingly a attractive target for the therapeutic intervention in cancer. Two FGFR inhibitors, namely AZD4547 and BGJ398, have entered phase II clinical trials, while more small-molecule inhibitors, such as SU5402, PD173074, TKI-258, and SU6668, have failed in clinical trials due to various complications associated with the side effects caused by the ATP-competitive inhibition mode [8]. To date, only five non-ATP-competitive FGFR inhibitors have been identified, including nordihydroguaiaretic acid (NDGA) [18], NF449 [19], ARQ069 [20], and recently reported A114 and A117 [21]. Despite advances in the field, identifying highly-selective, small-molecule inhibitors that target an inactive conformation or a new domain of FGFR continues to be a significant challenge.
In this report, we characterize two nordihydroguaiaretic acid (NDGA) analogs, i.e., Ad23 and Af23, as two kinase inhibitors that effectively target FGFR1 (Figure 1A). Furthermore, we provide data that indicates these kinase inhibitors have a distinct ATP-independent mode of action. Furthermore, these two compounds have shown excellent anti-cancer activity against NSCLC H460 cells both in vitro and in vivo. These data further confirm that bisaryl-1,4-dien-3-one structures could be used as nonATP-competitive FGFR1 inhibitors for the treatment of NSCLC.

\section{Methods}

\section{Cell lines, compounds, and reagents}

NSCLC cell line, H460, was purchased from ATCC (Manassas, VA). FGFR1-overexpressing HEK 293 cell lines were kindly gifted by the Institute of Materia Medical, Xi'an Jiaotong University. Various compounds were designed and synthesized in our laboratory, including Af23 (3,5-bis(3,4-dihydroxybenzylidene)-tetrahydropyran4-one) and $\operatorname{Ad} 23$ (3,5-bis(3,4-dihydroxybenzylidene)-tetrahydrothiopyran-4-one) (Figure 1A). Their purity was detected by HPLC $(>98.0 \%)$ before they were used in biological experiments. The compounds were dissolved in DMSO solution for in vitro assay. Their watersoluble formulations for in vivo studies were prepared

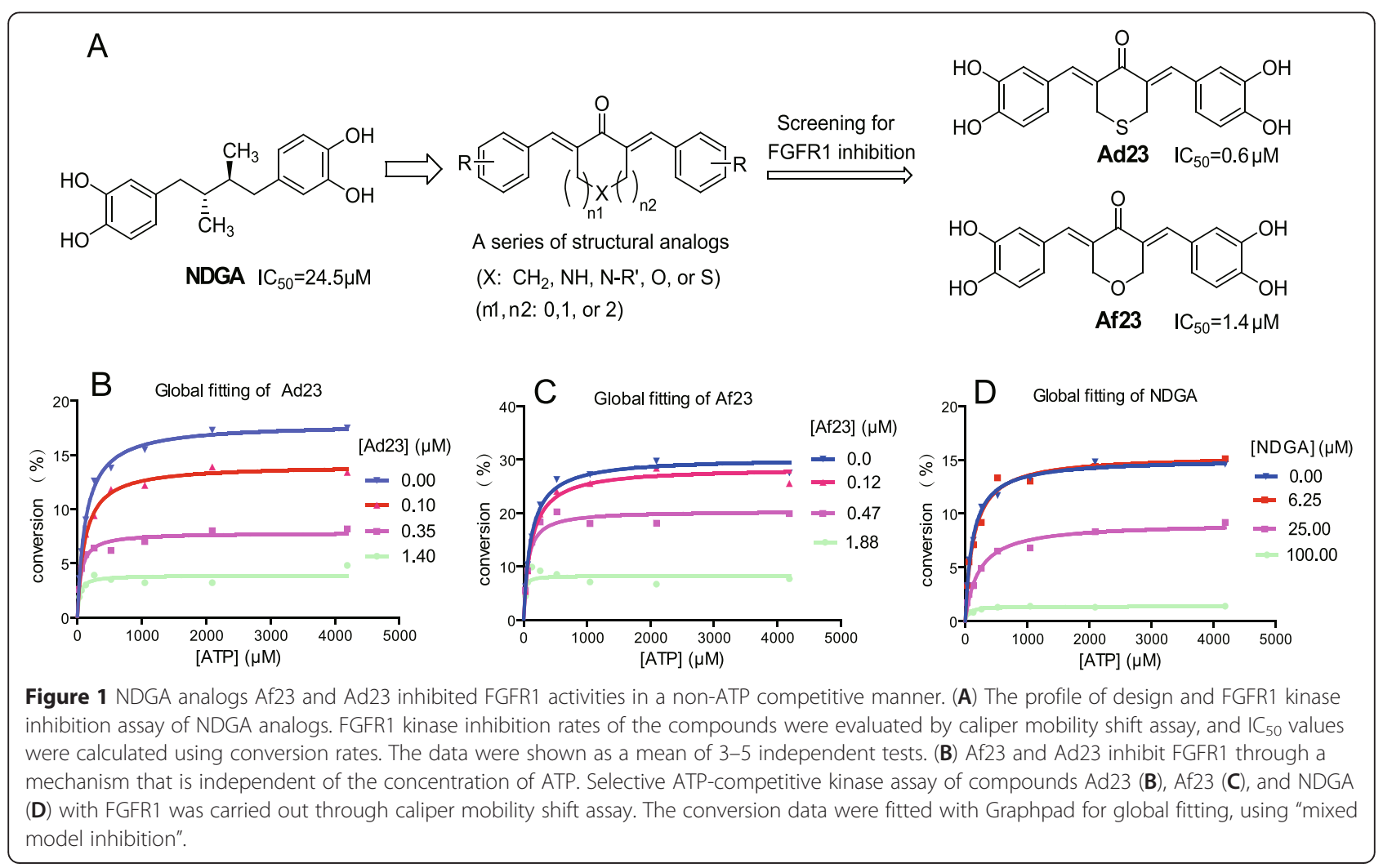


using the pharmaceutical method described previously [22]. All the antibodies were purchased from Santa Cruz Biotechnology (Santa Cruz, CA). Hoechst staining kit was purchased from Beyotime Biotech (Nantong, China). Recombinant FGFR1 proteins were obtained from Carna Biosciences, Inc. (Kobe, Japan).

\section{Cell-free FGFR1 kinase assays}

Using the method described previously in Ref. [21], the FGFR1 kinase inhibition assay of NDGA and its analogs were performed by Caliper Mobility Shift Assay with ATP concentration at its $\mathrm{Km}$ value $(262 \mu \mathrm{M})$. Staurosporine was used as a positive control. For the determination of $\mathrm{IC}_{50}$, the compounds were tested in duplicate at 10 concentrations, ranging from $5 \mathrm{nM}$ to $100 \mu \mathrm{M}$. Then, conversion data were collected on Caliper EZ reader (Hopkinton, $M A$ ). The $\mathrm{IC}_{50}$ values were obtained by GraphPad Prism 5 (GraphPad, San Diego, CA).

\section{ATP competitive inhibition assay}

This experiment was performed to test the relationship between the compounds and ATP in which the concentration of the substrate was constant, while the concentrations of ATP were set at 4192, 2096, 1048, 524, 262, 131,66 , and $33 \mu \mathrm{M}$. The global competitive inhibition fit for the compounds was performed based on percent conversion $=\left(\operatorname{Vmax}^{*} \mathrm{X}\right) /\left\{\mathrm{km}^{*}\left[(1+\mathrm{I} / \mathrm{Ki})^{\mathrm{n}}\right]+\mathrm{X}\right\}$, where $\mathrm{X}$ is the ATP concentration, and $\mathrm{n}$ is the Hill coefficient. Specific details of this method were presented in a previous report [21].

\section{MTT assay}

The anti-proliferative activities of compounds were detected by MTT assay. H460 cells were seeded in a 96well plate with RPMI-1640 medium that contained 0.1\% FBS for $24 \mathrm{~h}$. Then, the cells were treated with the compounds at the indicated concentrations $(0.74,2.22,6.67$, 20 , and $60 \mu \mathrm{M}$ ) for $72 \mathrm{~h}$. The proliferation of the $\mathrm{H} 460$ cells was detected through MTT assay, and the $\mathrm{IC}_{50}$ values were calculated by GraphPad software.

\section{Western blot analysis}

Cells or homogenated tumor tissues were lysated. Protein concentrations in all the samples were determined by using the Bradford protein assay kit (Bio-Rad, Hercules, $C A$ ). The lysates were separated by SDS-PAGE electrophoresis, and electro-transferred to a $0.22-\mu \mathrm{m}$ polyvinyldene difluoride membrane. After blocking with TBS that contained $5 \%$ non-fat milk for $1.5 \mathrm{~h}$ at room temperature, the membranes were incubated with different primary antibodies overnight at $4^{\circ} \mathrm{C}$. Following the TBST wash, immuno-reactive bands were detected by incubating with respective secondary antibody conjugated with horseradish peroxidase for $1 \mathrm{~h}$. Immuno- reactive bands were visualized by using an ECL kit (BioRad, Hercules, CA).

\section{Hoechest staining}

After the $\mathrm{H} 460$ cells were incubated with the compounds for $72 \mathrm{~h}$, cells were stained with Hoechst 33342 dye according to the protocol provided with the kit (Beyotime Biotech, Nantong, China). The cells were imaged under fluorescent microscope (Nikon, Tokyo, Japan), and the pictures were taken at $200 \times$ objective.

\section{Analysis of cell apoptosis}

H460 cells were placed in 60-mm plates for $12 \mathrm{~h}$ and then treated with NDGA, Ad23, or Af23 at the indicated concentrations for $24 \mathrm{~h}$. Then, the cells were harvested and stained with annexin $\mathrm{V}$ and propidium iodide (PI) in the presence of $100 \mathrm{mg} / \mathrm{mL}$ of RNAse and $0.1 \%$ Triton $\mathrm{X}-100$ for $30 \mathrm{~min}$ at $37^{\circ} \mathrm{C}$. Flow-cytometric analysis was performed using FACS calibur (BD Sciences, $C A$ ).

\section{In vivo anti-tumor study}

All animal experiments complied with the Wenzhou Medical College Policy on the Care and Use of Laboratory Animals (Wenzhou Medical College Animal Policy and Welfare Committee, Document ID: 201100103). Five to six-week-old athymic nu/nu female BALB/cA mice (18-22 g) were purchased from the Animal Center of the China Pharmaceutical University (Nanjing, China). The animals were housed at a constant room temperature with a 12:12-hr (light: dark) cycle and fed a standard rodent diet and water. H460 cells were harvested and mixed with Matrigel at proportions of 1:1. Then, the cells were injected subcutaneously into the right flank $\left(2 \times 10^{6}\right.$ cells in $200 \mu \mathrm{L}$ of PBS) of 7-week-old, BALB/cA nude mice. Two days after the $\mathrm{H} 460$ cells were injected, the mice were injected intraperitoneally (i.p.) with a water-soluble preparation of either compound Ad23 or compound Af23 in PBS at a dosage of $5 \mathrm{mg} / \mathrm{kg} /$ day for 28 days, whereas the control mice were injected with the liposome vehicle in PBS ( $n=10$ in each group). The volume of the tumors were determined by measuring their length (l) and width (w) and calculated using the formula; $\mathrm{V}=0.52 \times 1 \times \mathrm{w}^{2}$. The weight of the tumors were recorded on the day the mice were killed.

\section{Immunohistochemistry analysis}

On day 30 after tumor induction, the mice were killed in a $\mathrm{CO}_{2}$ chamber, and the tumor tissues were dissected and weighed. Some of the tissues were lysed for protein isolation and then processed for the determination of signaling pathway proteins using Western blot method. A part of harvested tumor tissues were fixed in $10 \%$ formalin at room temperature overnight, processed, and embedded in paraffin. The paraffin-embedded tissues 
were sectioned (5- $\mu \mathrm{m}$ thick) followed by staining with primary antibodies. The signal was detected by biotinylated secondary antibody and developed with 3,3-diaminobenzidine $(\mathrm{DAB})$.

\section{Statistical analysis}

All in vitro experiments were repeated at least three times. Data were presented as means \pm SD or mean \pm SEM. The statistical significance of differences between groups was obtained by the student's $t$ test or ANOVA multiple comparisons in GraphPad Prism 5 (License Number: GPW5-415777-RAG-2191, GraphPad Software Inc., San Diego, CA). $P$ values less than $0.05(p<0.05)$ were considered to be significant.

\section{Results}

\section{Af23 and Ad23 inhibits FGFR1 kinase via a non-ATP} dependent manner

The leading compound NDGA is a natural product isolated from creosote bush. It exhibits multiple pharmacological effects, such as anti-oxidation, anti-inflammation, and anti-tumor $[18,23]$. Recently, Meyer et al. found that NDGA could inhibit the autophosphorylation of FGFR3 kinase both in vitro and in vivo [18]. In our previous cell-free assay, we found that the $\mathrm{IC}_{50}$ values of NDGA against FGFR1 and FGFR3 were 24.5 and $72.4 \mu \mathrm{M}$, respectively, indicating that NDGA exhibits better inhibitory activity against FGFR1 than FGFR3 (Figure 1A). Therefore, using NDGA as a leading compound, we designed and synthesized a series of structural analogs (Figure 1A). Next, we tested the inhibitory activity of synthetic NDGA analogs against FGFR1 kinase by mobility shift assay.

The inhibitory potency of 72 bisaryl-1,4-dien-3-one compounds against FGFR1 kinase was evaluated by in vitro kinase assays. Out of the 72 compounds, Ad23 and Af23 were found to exhibit much stronger inhibition against FGFR1 kinase activity than NDGA and other analogs $\left(\mathrm{IC}_{50}\right.$ : Ad23,0.6 $\mu \mathrm{M}$; Af23,1.4 $\mu \mathrm{M}$ ) (Figure 1A). Thus these two were chosen for further studies. Subsequently, the kinase inhibition modes of both $\operatorname{Ad} 23$ and Af23 were studied. As shown in Figure 1B, the velocity of FGFR1 substrate phosphorylation without inhibitors increases as the ATP concentration increased, and it was reached to the peak at an ATP concentration of $2000 \mu \mathrm{M}$. At concentrations greater than the $\mathrm{IC}_{50}$ value (1.4 $\mu \mathrm{M}$ for Ad23; $1.88 \mu \mathrm{M}$ for Af23; $100 \mu \mathrm{M}$ for NDGA), the kinase activity was decreased by more than $90 \%$, and further increases in the concentration of ATP, even up to $4190 \mu \mathrm{M}$, had no effect on the inhibitory potency of the compounds (Figure 1B). These results showed that the inhibition of FGFR1 kinase activity by Ad23, Af23, and NDGA was not dependent on the concentration of ATP. Thus, we obtained two novel non-
ATP-competitive FGFR1 inhibitors, i.e., Ad23 and Af23, from the leading NDGA.

\section{Ad23 and Af23 inhibits the cellular FGFR1 phosphorylation}

The inhibitory effects of these two compounds on FGFR1 activation were determined in FGFR1-overexpressing 293 cells and human NSCLC H460 cells. As shown in Figures $2 \mathrm{~A}$ and $\mathrm{B}$, pre-treatment with $\mathrm{Ad} 23$ or Af23 dose-dependently reduced the bFGF-induced phosphorylation of FGFR1 in both the cell lines. Also, both Ad23 and Af23 inhibited the phosphorylation of FRS2, a proliferative substrate of FGFR1, in a dose-dependent manner in $\mathrm{H} 460$ cells (Figure 2C). Consistent with the cell-free results, Ad23 and Af23 had greater activity than NDGA, and Ad23 showed stronger inhibition than Af23 against cellular FGFR1 phosphorylation.

\section{Ad23 and Af23 inhibited the proliferation and induced the apoptosis of $\mathrm{H} 460$ cell line}

Next, the growth inhibition of H460 cells by NDGA, Ad23, and Af23 was studied by MTT assay. Our data showed that Af23, Ad23, and NDGA exhibited marked inhibitory effects against $\mathrm{H} 460$ cells (Figure 3A). The inhibitory potencies ( $\mathrm{IC}_{50}$ values) of Af23 and $\mathrm{Ad} 23$ were much greater when compared to NDGA. Further, Western blot analysis showed that the cleavage of caspase- 3 and caspase-9 increased after treatment with $\operatorname{Ad} 23$ or Af23, indicating that these compounds could induce apoptosis in H460 cells after a 12-h treatment (Figure 3B). Further, Hoechst staining was performed $12 \mathrm{~h}$ after treatment with the compounds (Figure 3C). A concentration-dependent increase in the number of cells with nuclear condensation and fragmentation was observed in both the groups. Next, we assessed the effects of Ad23 and Af23 on the induction of apoptosis in $\mathrm{H} 460$ cells by flow cytometric analysis. Figure 3D shows that both $\operatorname{Ad} 23$ and Af23 dosedependently increased the H460 apoptosis after 24-h treatment. At a concentrations of $20 \mu \mathrm{M}$, both Ad23-treated group (Annexin $\mathrm{V}^{+} / \mathrm{PI}^{+}, 44.8 \pm 7.07 \%$ ) and Af23-treated group $(48.2 \pm 11.12 \%)$ induced a greater rate of cell apoptosis than NDGA (27.2 $\pm 5.27 \%)$. We have also tested the growth inhibition of Ad23 and Af23 against human liver cells HL7702 and human fetal lung cell line MRC-5 which expresses low level of FGFR1. The results showed that all the $\mathrm{IC}_{50}$ values of Ad23, Af23, and NDGA against HL7702 or MRC-5 cells were greater than $30 \mu \mathrm{M}$ (Figure 3A).

\section{Ad23 and Af23 significantly suppressed the $\mathrm{H} 460$ tumor growth in xenograft mouse model}

In order to further assess the anti-tumor activities of the NDGA analogs, we tested the efficacies of Af23 and Ad23 in the H460 xenograft mouse model. Treatment with Af23 or Ad23 for 28 days resulted in significant 


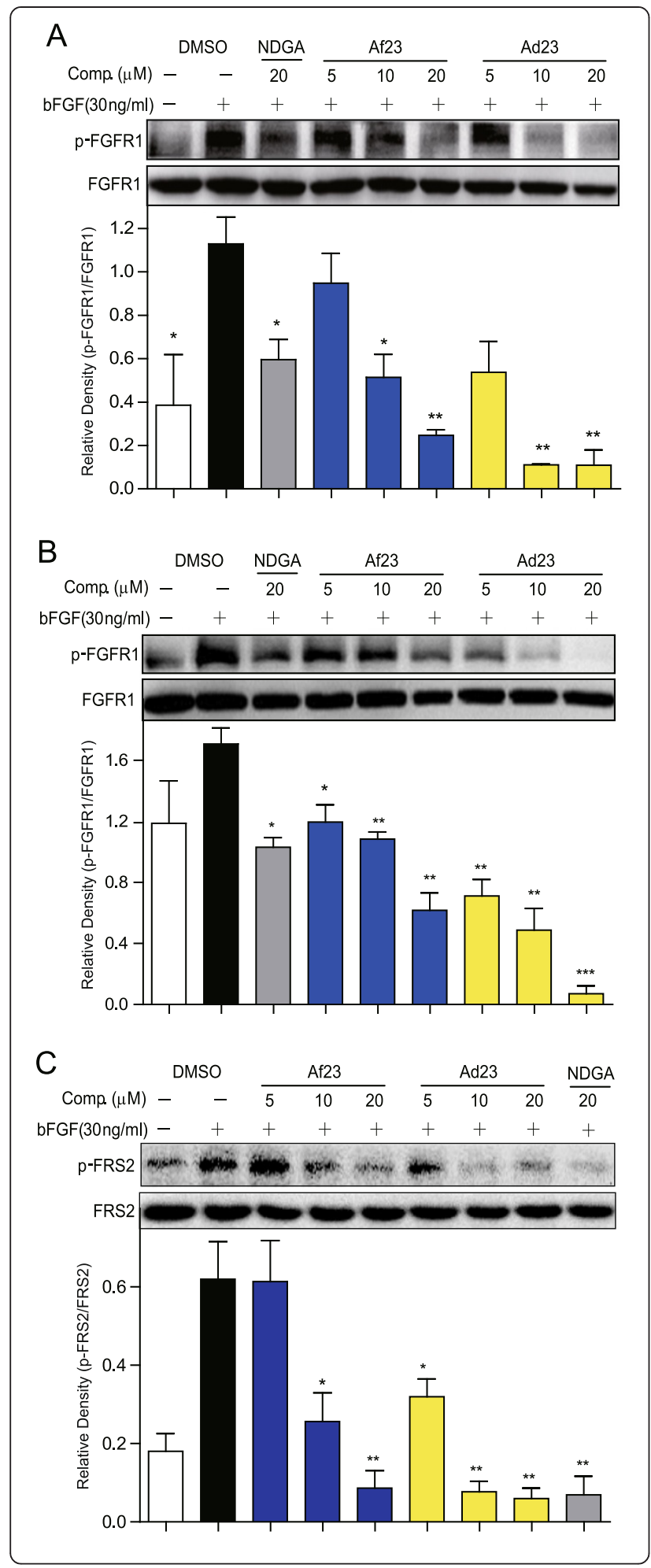

Figure 2 Compounds Ad23 and Af23 inhibited intracellular FGFR1/ FRS2 phosphorylation. FGFR1 over-expression 293 cells (A) or H460 cells (B and $\mathbf{C}$ ) were pretreated with compounds at indicated concentrations or vehicle ( $0.1 \% \mathrm{DMSO})$, respectively. Then, cells were stimulated with bFGF (30 ng/mL) for $10 \mathrm{~min}$, and the phosphorylation levels of FGFR1 (A and $\mathbf{B}$ ) and FRS2 (C) in cell lysates was measured by western blot analysis. The figures were representative of 3 separate experiments. The column figures show the normalized optical density as a percentage of total protein control. Bars represent the mean \pm SEM of 3 independent experiments. Statistical significance relative to bFGF alone group was expressed, ${ }^{*} P<0.05$, ${ }^{* *} P<0.01$, ${ }^{* * *} P<0.001$.

reduction in tumor volume (Figure 4A). The weight of the tumors were also reduced markedly in the Af23treated and Ad23-treated groups, with the inhibition rate of 67.4 and $75.8 \%$, respectively (Figure $4 \mathrm{~B}$ ). To evaluate whether the inhibition of tumor growth by Ad23 or Af23 was associated with the inhibition of FGFR1 activity in vivo, we analyzed the expression of p-FGFR1 in the tumor tissues. As shown in Figure 4C, Ad23 and Af23 exhibited a significant increased inhibitory effect on FGFR1 phosphorylation in H460 tumor xenografts than the vehicle control tumors. Also, no obvious toxicity was observed in the Af23-treated group or the Ad23-treated group, evidenced by no obvious loss of weight among the two groups during the period of treatment (Figure 4D).

Prior studies have revealed that phosphorylation of FGFR1 can lead to the activation of downstream signaling cascades including ERK and AKT, which plays an important role in the proliferation and survival of cancer cells. Thus, the phosphorylation of ERK and AKT in tumor samples was tested by immunohistochemical assays (Figure 4E). Similarly, these compounds inhibited the FGFR1-downstream ERK and AKT phosphorylation. Finally, the immunochemical data also showed that the expression of Bcl-2, Cyclin D1 and COX-2 was reduced to a far greater extent in the Ad23-treated group and the Af23-treated group than compared to the vehicle-treated group (Figure 4E), indicating that these two FGFR1 inhibitors also induced tumor-cell apoptosis in H460 xenografts.

\section{Discussion}

FGFR1 is highly related to the development of lung cancer $[10,12-15,17,24]$. Ren et al. identified four NSCLC cell lines and two, newly-established primary lungcancer cultures that showed high FGFR1 expression levels [24]. They also found that treatment with ponatinib, an FGFR1 inhibitor, could inhibit cell growth in NSCLC cell lines [24]. Terai et al. revealed that the activation of the FGF2-FGFR1 autocrine pathway may be a novel mechanism of acquired resistance to the EGFR inhibitor, gefitinib, in NSCLC [12]. There has been increasing evidence indicating that FGFR1 inhibitors could 
A

\begin{tabular}{lcll}
\hline Comp. & H460 & HL7702 & MRC-5 \\
\hline Af23 & $9.9 \pm 1.2$ & $87.8 \pm 18.2$ & $33.6 \pm 5.1$ \\
Ad23 & $6.7 \pm 0.9$ & $>100$ & $54.7 \pm 6.9$ \\
NDGA & $15.8 \pm 1.2$ & $>100$ & $32.2 \pm 7.4$ \\
\hline
\end{tabular}

B

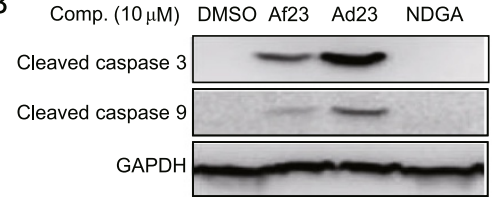

C
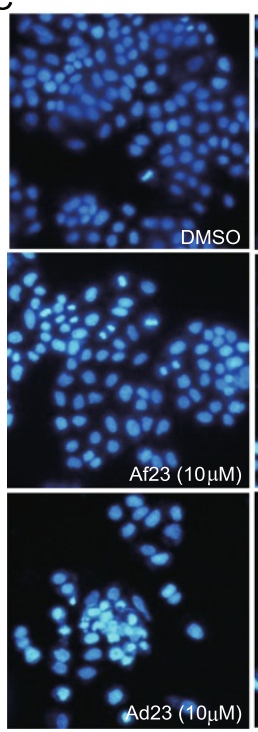
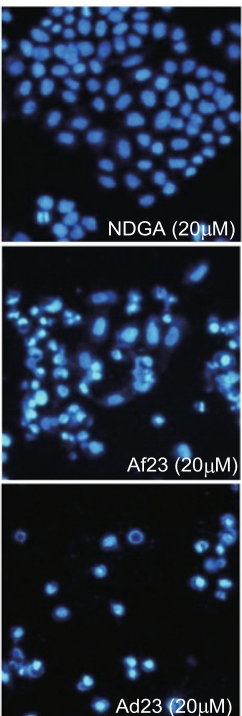

D

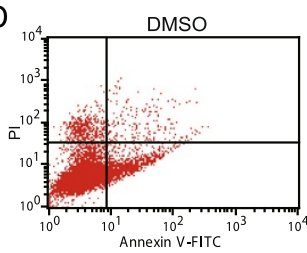

Af23 $(10 \mu \mathrm{M})$

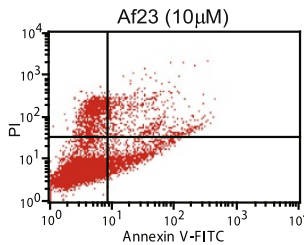

$\operatorname{Ad} 23(10 \mu \mathrm{M})$

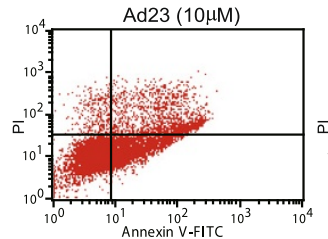

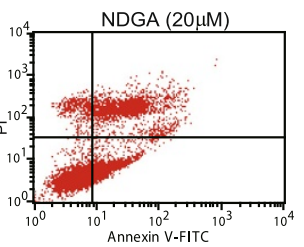

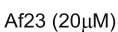

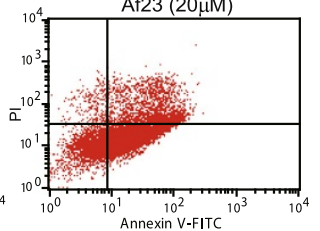

Ad23 $(20 \mu \mathrm{M})$

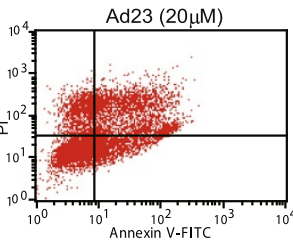

Figure 3 Af23 and Ad23 inhibited proliferation and induced apoptosis in H460 cells. (A) H460 cells were treated with Af23, Ad23 or NDGA at different concentration $(0.74,2.22,6.67,20$, and $60 \mu \mathrm{M})$ for $72 \mathrm{~h}$. The viability of $\mathrm{H} 460$ cells were detected by MTT assay, and the $I \mathrm{C}_{50}$ values of compounds were fitted with GraphPad. (B) Effects of Af23 and Ad23 on caspase activation in H460 cells. H460 cells were harvested and lysated after incubated with Af23 $(10 \mu \mathrm{M})$, Ad23 $(10 \mu \mathrm{M})$, or NDGA $(10 \mu \mathrm{M})$ for $12 \mathrm{~h}$. The levels of cleaved caspase-3 and cleaved caspase-9 were determined by western blot analysis. (C) Morphological changes and hoechst staining were observed in $\mathrm{H} 460$ cells cultured with and without Af23, Ad23, or NDGA at indicated concentrations for $12 \mathrm{~h}(200 \times)$. The figures were representative of more than three separate experiments. D. Af23 and Ad23 induced cell apoptosis in H460 cells. H460 cells were treated with Af23, Ad23, or NDGA at indicated concentrations for 24 h, and then stained with Annexin $\mathrm{V}$ and PI, followed by detection using flow cytometry. The representative pictures are shown.

be a promising candidate for the treatment of NSCLC in clinics. Although a variety of ATP-competitive FGFR1 inhibitors with therapeutic prospects for lung cancer have been identified, most of them failed in pre-clinical or clinical studies because of their low efficacy or high toxicity. At present only two selective FGFR1 inhibitors, i.e., AZD4547 and BGJ398, are being studied in clinical trials (phase II) [8]. The ATP-competitive FGFR1 inhibitors functions by targeting the ATP-binding pocket of FGFR1, which may lead to a decrease in their efficiency when high physiological or intracellular concentrations of ATP exist [8]. In addition, since the ATP-binding site is highly conserved in RTKs, most inhibitors exhibit limited selectivity within RTKs, which induces side effects during treatment, such as nausea, weakness, and elevated blood pressure [25]. For instance, PD173074 and SU5402 failed to enter phase II clinical trials due to their high toxicities [25]. Therefore, the exploration of nonATP-competitive FGFR1 inhibitors has attracted extensive attention in recent years.
NDGA was reported previously to inhibit FGFR3 kinase [18]. In this study, we found that NDGA exhibited better inhibitory activity against FGFR1 than FGFR3. Using NDGA as a leading compound, we designed and synthesized several NDGA analogs with the basic skeleton of bisaryl-1,4-dien-3-one (Figure 1A). After screening for kinase inhibition, we obtained two FGFR1 inhibitors (Ad23 and Af23) that had inhibitory activities better than compared to NDGA. Interestingly, NDGA and its analogs retained their potency for the inhibition of kinase activity when the concentration of ATP increased, suggesting that the inhibitory effects of these compounds were independent of the concentration of ATP (Figure 1B). So far, ARQ069 is the only published molecule that inhibits FGFR1/2 in a manner that does not compete with ATP. ARQ069 exhibits FGFR1/2 kinase inhibition with $\mathrm{IC}_{50}$ values of 0.84 and $1.23 \mu \mathrm{M}$, respectively [20]. Although the $\mathrm{IC}_{50}$ values of $\mathrm{Ad} 23$, Af23, and ARQ069 are at micromolar concentration levels, the observation that the inhibition of FGFR1 autophosphorylation 


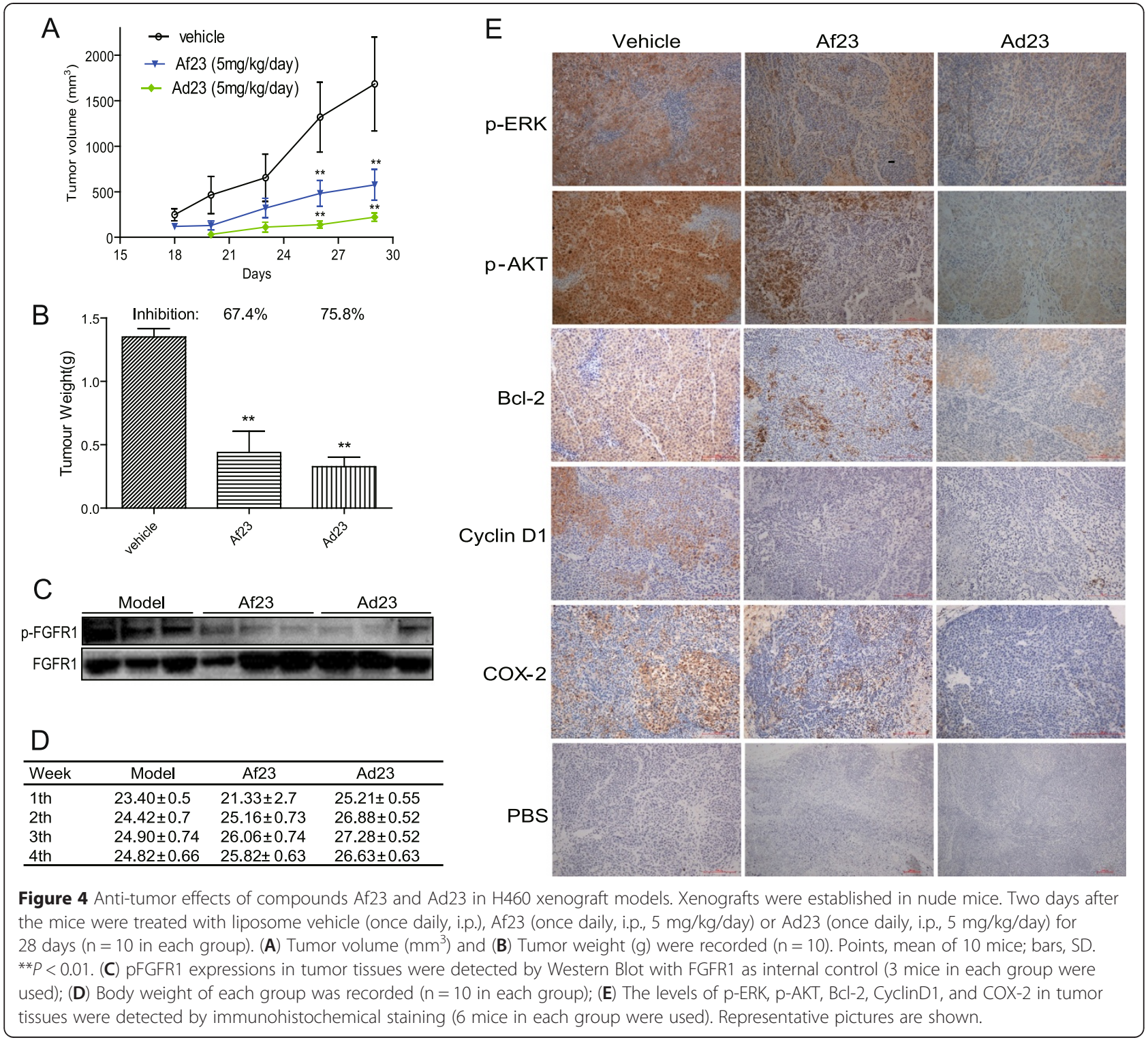

(Figure 2) and the inhibition of the direct FGFR1 downstream substrate (Figure 1A) occur at different dose levels was consistently detectable. A literature search on kinase inhibitors yielded several reports describing that nonATP-competitive kinase inhibitors may not display identical $\mathrm{IC}_{50}$ values with that of classic ATP-competitive inhibitors for the inhibition of both kinase autophosphorylation and downstream signaling pathways $[20,24]$. Our novel NDGA analogs (Ad23 and Af23) also showed micromole-grade FGFR1 inhibitory effects in a manner that was independent of ATP.

Figure 2 further revealed the anti-FGFR1 ability of Ad23 and Af23 in the cellular levels using FGFR1overexpressed 293 cells and NSCLC H460 cells. These two compounds also dose-dependently inhibited FRS2 phosphorylation in $\mathrm{H} 460$ cells. These data led us to investigate the anti-cancer efficacy of Ad23 and Af23 further. We evaluated the anti-proliferative effects of Ad23 and Af23 in vitro. As shown in Figure 3, Ad23 and Af23, as well as NDGA, inhibited cell growth and induced cell apoptosis in NSCLC H460 cells; they also had low toxicities against normal MRC- 5 cells and normal human liver cells (HL-7702 cells). Further, we showed the potent anti-tumor ability of these two inhibitors in the H460 xenograft mouse model. Previously, studies on ARQ069, a non-ATP-competitive inhibitor, did not report the in vivo anti-tumor activity. In the present study, the data revealed that the compounds Ad23 and Af23 suppressed the phosphorylation of FGFR1 in H460 tumor tissues, thereby inhibited the downstream phosphorylation of ERK and AKT, and reduced the expression of BCL-2, COX-2, and Cyclin D1 (Figure 4). At the 
same time, we observed that Ad23 and Af23 exhibited high safety in vivo (Figure 4D). As already known, the ATP-competitive inhibitory mode leads to the biggest problems (toxicity and side effects) of current FGFR1 inhibitors. Our findings for Ad23 and Af23 indicated that the non-ATP-competitive FGFR1 inhibition might be a new cancer therapeutic alternative with much lower toxicity in vivo. However, continued research is needed to examine the underlying FGFR1-binding mechanism and preclinical evaluation of these two compounds. In spite of the predicted "DFG-OUT" docking model, it is unclear how Ad23/Af23 exactly binds to the FGFR1 kinase domain or other domains, which needs to be demonstrated by X-ray diffraction-based structural biology study. Also, it is important to test the RTK-inhibitory selectivity of these two compounds at both molecular and cellular levels in the future.

\section{Conclusion}

Given the critical importance of FGFR1 in the pathogenesis and development of NSCLC, this study identified two novel, non-ATP-competitive inhibitors of FGFR1 kinase, i.e., Ad23 and Af23, both of which exhibited good anti-tumor activity in vitro and in vivo. These two compounds were shown to have the potential to be developed as novel agents for the treatment of NSCLC. The results of the present study indicate that $\operatorname{Ad} 23$ and Af23deserve further studies both in the pre-clinical evaluation and in the field of medicinal chemistry for developing structurally different and more effective bisaryl1,4-dien-3-one-containing FGFR1 inhibitors.

\section{Competing interests}

The authors declare that they have no competing interests.

\section{Authors' contributions}

YC and JW analyzed data and wrote the paper; JW, TW, QT, BW, XJ, and WL performed experiments; $\mathrm{GL}, \mathrm{XL}$, and JJ designed the research project and experiments, $J J$ and $Y C$ analyzed data and revised the paper; TD and GL revised the paper. All authors read and approved the final manuscript.

\section{Acknowledgements}

The work was supported by National Natural Science Foundation of China (81173083, 81272462, and 81473242), Natural Science Funding of Zhejiang Province (LY13H160022, LY12H16003, and LY12H30005), Key Science Funding of Zhejiang Department of Health (WKJ2013-2-021), and Zhejiang Key Group Project in Scientific Innovation (2010R50042).

\section{Author details}

${ }^{1}$ Chemical Biology Research Center, College of Pharmaceutical Sciences, Wenzhou Medical University, Wenzhou, Zhejiang 325035, China. ${ }^{2}$ College of Information Science and Computer Engineering, Wenzhou Medical Universtiy, Wenzhou, Zhejiang 325035, China. ' ${ }^{3}$ Department of Pharmacy, the Sixth Affiliated Hospital of Wenzhou Medical University, Lishui, Zhejiang 323000, China. ${ }^{4}$ Department of Interventional Radiology, The Fifth Affiliated Hospital of Wenzhou Medical University, Lishui, Zhejiang 323000, China.

Received: 4 June 2014 Accepted: 31 March 2015

Published online: 12 April 2015

\section{References}

1. Kono SA, Marshall ME, Ware KE, Heasley LE. The fibroblast growth factor receptor signaling pathway as a mediator of intrinsic resistance to EGFRspecific tyrosine kinase inhibitors in non-small cell lung cancer. Drug Resist Updat. 2009;12(4-5):95-102.

2. Saijo N. Targeted therapies: Tyrosine-kinase inhibitors-new standard for NSCLC therapy. Nat Rev Clin Oncol. 2010;7(11):618-9.

3. Sordella R, Bell DW, Haber DA, Settleman J. Gefitinib-sensitizing EGFR mutations in lung cancer activate anti-apoptotic pathways. Science. 2004;305(5687):1163-7.

4. Horn L, Sandler A. Epidermal growth factor receptor inhibitors and antiangiogenic agents for the treatment of non-small cell lung cancer. Clin Cancer Res. 2009;15(16):5040-8.

5. Zhou W, Ercan D, Chen L, Yun CH, Li D, Capelletti M, et al. Novel mutantselective EGFR kinase inhibitors against EGFR T790M. Nature. 2009:462(7276):1070-4.

6. Hammerman PS, Janne PA, Johnson BE. Resistance to Epidermal Growth Factor Receptor Tyrosine Kinase Inhibitors in Non-Small Cell Lung Cancer. Clin Cancer Res. 2009;15(24):7502-9.

7. Zhang J, Yang PL, Gray NS. Targeting cancer with small molecule kinase inhibitors. Nat Rev Cancer. 2009:9(1):28-39.

8. Liang G, Liu Z, Wu J, Cai Y, Li X. Anticancer molecules targeting fibroblast growth factor receptors. Trends Pharmacol Sci. 2012;33(10):531-41.

9. Fang Z, Grutter C, Rauh D. Strategies for the selective regulation of kinases with allosteric modulators: exploiting exclusive structural features. ACS Chem Biol. 2013;8(1):58-70.

10. Kohler LH, Mireskandari M, Knosel T, Altendorf-Hofmann A, Kunze A, Schmidt A, et al. FGFR1 expression and gene copy numbers in human lung cancer. Virchows Arch. 2012;461(1):49-57.

11. Dutt A, Ramos AH, Hammerman PS, Mermel C, Cho J, Sharifnia T, et al. Inhibitor-sensitive FGFR1 amplification in human non-small cell lung cancer. PLoS One. 2011;6(6):e20351.

12. Terai H, Soejima $K$, Yasuda H, Nakayama S, Hamamoto J, Arai D, et al. Activation of the FGF2-FGFR1 autocrine pathway: a novel mechanism of acquired resistance to gefitinib in NSCLC. Mol Cancer Res. 2013;11(7):759-67.

13. Tran TN, Selinger Cl, Kohonen-Corish MR, McCaughan BC, Kennedy CW, OToole SA, et al. Fibroblast growth factor receptor 1 (FGFR1) copy number is an independent prognostic factor in non-small cell lung cancer. Lung Cancer. 2013;81(3):462-7.

14. Preusser M, Berghoff AS, Berger W, Ilhan-Mutlu A, Dinhof C, Widhalm G, et al. High rate of FGFR1 amplifications in brain metastases of squamous and non-squamous lung cancer. Lung Cancer. 2014;83(1):83-9.

15. Heist RS, Mino-Kenudson M, Sequist LV, Tammireddy S, Morrissey L, Christiani DC, et al. FGFR1 amplification in squamous cell carcinoma of the lung. J Thorac Oncol. 2012;7(12):1775-80.

16. Yang J, Zhao H, Xin Y, Fan L. MicroRNA-198 inhibits proliferation and induces apoptosis of lung cancer cells via targeting FGFR1. J Cell Biochem. 2013. doi:10.1002/jcb.24742.

17. Zhang J, Zhang L, Su X, Li M, Xie L, Malchers F, et al. Translating the therapeutic potential of AZD4547 in FGFR1-amplified non-small cell lung cancer through the use of patient-derived tumor xenograft models. Clin Cancer Res. 2012;18(24):6658-67.

18. Meyer AN, McAndrew CW, Donoghue DJ. Nordihydroguaiaretic acid inhibits an activated fibroblast growth factor receptor 3 mutant and blocks downstream signaling in multiple myeloma cells. Cancer Res. 2008:68(18):7362-70.

19. Krejci P, Murakami S, Prochazkova J, Trantirek L, Chlebova K, Ouyang Z, et al. NF449 is a novel inhibitor of fibroblast growth factor receptor 3 (FGFR3) signaling active in chondrocytes and multiple myeloma cells. J Biol Chem. 2010;285(27):20644-53

20. Eathiraj S, Palma R, Hirschi M, Volckova E, Nakuci E, Castro J, et al. A novel mode of protein kinase inhibition exploiting hydrophobic motifs of autoinhibited kinases: discovery of ATP-independent inhibitors of fibroblast growth factor receptor. J Biol Chem. 2011;286(23):20677-87.

21. Wang Y, Cai Y, Ji J, Liu Z, Zhao C, Zhao Y, et al. Discovery and identification of new non-ATP competitive FGFR1 inhibitors with therapeutic potential on non-small-cell lung cancer. Cancer Lett. 2014;344:82-9.

22. Wu J, Li J, Cai Y, Pan Y, Ye F, Zhang Y, et al. Evaluation and discovery of novel synthetic chalcone derivatives as anti-inflammatory agents. J Med Chem. 2011;54(23):8110-23. 
23. Lu JM, Nurko J, Weakley SM, Jiang J, Kougias P, Lin PH, et al. Molecular mechanisms and clinical applications of nordihydroguaiaretic acid (NDGA) and its derivatives: an update. Med Sci Monit. 2010;16(5):RA93-100.

24. Ren $\mathrm{M}$, Hong $\mathrm{M}$, Liu G, Wang $\mathrm{H}$, Patel $\mathrm{V}$, Biddinger $\mathrm{P}$, et al. Novel FGFR inhibitor ponatinib suppresses the growth of non-small cell lung cancer cells overexpressing FGFR1. Oncol Rep. 2013;29(6):2181-90.

25. Liang G, Chen G, Wei X, Zhao Y, Li X. Small molecule inhibition of fibroblast growth factor receptors in cancer. Cyto Growth Factor Rev. 2013;24(5):467-75.

Submit your next manuscript to BioMed Central and take full advantage of:

- Convenient online submission

- Thorough peer review

- No space constraints or color figure charges

- Immediate publication on acceptance

- Inclusion in PubMed, CAS, Scopus and Google Scholar

- Research which is freely available for redistribution 